\title{
Implementation of the ELECTRE Method for Determining the Location of Evacuation of Web-based Tsunami Disaster
}

\author{
Toar Romario Sigar \\ Department of Information System \\ Diponegoro University \\ Semarang - Indonesia
}

\author{
Vincencius Gunawan \\ Department of Physics \\ Diponegoro University \\ Semarang - Indonesia
}

\author{
Jatmiko Endro Suseno \\ Department of Physics \\ Diponegoro University \\ Semarang - Indonesia
}

\begin{abstract}
The selection of good tsunami evacuation sites is one of the factors that influence the disaster mitigation process. Method ELimination Et Choix TRadusiant la REalite (ELECTRE) is one of the models that can be used to determine the best alternative of selected alternatives. By using the ELECTRE method it can determine the location of tsunami disaster evacuation in accordance with the criteria that have been determined by the Regional Disaster Management Agency of Bitung City. In this study will conduct an analysis to prove that the ELECTRE method can be used to determine the location of tsunami disaster evacuation from a number of alternative locations with predetermined criteria and thereby get the results of the analysis and calculation using the ELECTRE method resulted in the location of SMP Negeri 12 Bitung with has the total value of $\mathbf{E}_{\mathrm{kl}}=1$ of 6 , then according to the conclusion below the location serve as the best location to be the location of the tsunami evacuation point based on the criteria given by the stakeholders in this case Regional Disaster Management Agency Bitung.
\end{abstract}

\section{General Terms}

Method ELECTRE

\section{Keywords}

Method ELECTRE, Tsunami Disaster, Evacuation Location

\section{INTRODUCTION}

Indonesia is an area prone to earthquake and tsunami disaster because Indonesia topographic location is located at the meeting of 3 main tectonic plates of the world. The three plates are the Indian Ocean Plate - Australia to the south of the island of Java and west of the island of Sumatra, the Pacific Ocean Plate is to the east, the northern Eurasian Plate (which mostly enters the Indonesian territory), plus the Sea Plate The Philippines is just adjacent to North Sulawesi [1]. The earthquake that followed the great Tsunami in Aceh in 2014 has resulted in enormous social, economic, physical and environmental losses. Most of the victims were communities in coastal and lowland areas without knowledge of tsunami hazards and their impacts. Therefore, to reduce the risks posed by the tsunami hazard, the level of community vulnerability must be reduced by improving human resources capacity and applying good early evacuation and rank system (Regional Disaster Management Agency Bitung City).

In this study will use the method of ELECTRE and research research conducted with the method of ELECTRE such as, Selection of contract outsourcing [2], Implementation in the energy sector [3], Implementation in tourism sector [4], Comparing Analytic Hierarchy Process (AHP) and ELECTRE to determine the priority requirements of a software [5], The application of ELECTRE to analyze the operational performance comparison of mobile service provider providers [6], Application of the ELECTRE method for supplier selection process [7], Application of the ELECTRE method for the recommendation of the Study Program [8].

The advantages of the ELECTRE method can be applied with more alternate conditions than the number of criteria, so the ELECTRE method with the concept of outranking relation alone can identify the choice between two alternatives. If one alternative will outperform the other alternatives only if there are sufficient conditions to convince below one alternative is better than the other alternative or at least one alternative is as good as the other alternative, making the process faster. [9], [10].

Based on the above problems and the advantages of the ELECTRE method itself will be built a web-based decision support system that will assist in the process of selecting locations where the tsunami disaster evacuation. The location of the disaster evacuation site is highly dependent on parameters such as Location, Population, Accessibility, Topographic Area, Building Orientation, and Space Utilization referring to the Regulation of the Head of National Disaster Management Agency Number 14 of 2010 and data issued by the Regional Disaster Management Agency (BPBD) city of Bitung. Because this is because the city of Bitung is one of the disaster-prone areas of the tsunami, based on the results of the preparation of the Plan for Earthquake and Tsunami Disaster BPBD Bitung City in 2016, has set the areas that can be used as a disaster evacuation space and the establishment of evacuation path that will leading to a safe location.

\section{METHOD ELECTRE}

Method ELimination Et Choix Tradusiant la REalite or in English meaning Elimination and Choice Expressing Reality (ELECTRE) is one of the multicriteria decision-making methods developed in 1960 by Bernard Roy. The ELECTRE method involves a systematic analysis of the relationship between all possible different pairwise options, based on the value of each option on a common set of evaluation criteria and the result is a measure of the extent to which each option outperforms everything else. The basic concept of the ELECTRE method is to handle outranking relations by using pairwise comparisons between alternatives based on each separate criterion [11]. Outranking relation itself is a relationship to identify the choice between two alternatives. One alternative outperforms the other alternatives, only if there are sufficient conditions to convince below one 
alternative to the other alternatives or at least one alternative as good as any other alternative [9]. The steps taken in various problems using ELECTRE are as follows [12]:

Step 1: Normalize the decision matrix.

Normalization of the matrix $\boldsymbol{r}_{i j}$ can be done with the following equation:

$\boldsymbol{r}_{i j}=\frac{\boldsymbol{x}_{i j}}{\sqrt{\sum_{i=1}^{m} x_{i j}^{2}}}$, for $\mathrm{i}=1,2,3, \ldots \mathrm{m}$ and $\mathrm{j}=1,2,3, \ldots \mathrm{n}$

then obtained matrix $\boldsymbol{R}$ result of normalization

$r=\left[\begin{array}{llll}r_{11} & r_{12} & \cdots & r_{1 n} \\ r_{21} & r_{22} & \cdots & r_{2 n} \\ r_{m 1} & r_{m 2} & \cdots & r_{m n}\end{array}\right]$

$\boldsymbol{R}$ is a normalized matrix called normalized decision matrix. With $m$ declare an alternative, $\boldsymbol{n}$ states the criteria dan $\boldsymbol{r}_{i j}$ a measurement of choice from alternative to- $\boldsymbol{i}$ in relation to the criterion to-j.

Step 2: Weighing on a normalized matrix.

After normalization, each column of the matrix $\boldsymbol{R}$ is multiplied by the weight of the criterion with the following equation:

$v_{i j}=w_{i} r_{i j}$

Step 3: Determine the set of concordance and discordance index

For each pair of alternatives $k$ and $l(k, l=1,2,3, \ldots, \mathrm{m}$ and $\mathrm{k}$ $\neq 1$ ) set of criteria $j$ divided into two subsets, namely concordance and discordance. When a criterion in an alternative includes concordance in the following equation:

$\boldsymbol{c}_{\boldsymbol{k} \boldsymbol{l}}=\left\{\boldsymbol{j} \mid \boldsymbol{y}_{\boldsymbol{k} j} \leq \boldsymbol{y}_{\boldsymbol{l} \boldsymbol{j}}\right\}$, for $j=1,2,3, \ldots, \mathrm{n}$

In contrast, the complementary of this subset is discordance, ie when:

$\boldsymbol{d}_{\boldsymbol{k} \boldsymbol{l}}=\left\{\boldsymbol{j} \mid \boldsymbol{y}_{\boldsymbol{k} \boldsymbol{j}}<\boldsymbol{y}_{\boldsymbol{l} \boldsymbol{j}}\right\}$, for $j=1,2,3, \ldots, \mathrm{n}$.

Step 4: Calculate concordance and discordance matrices

\section{Concordance}

To determine the value of the elements in the concordance matrix is to add the weights included in the concordance subset by using the following equation:

$c_{k l}=\sum_{j \in C_{k l}} W_{j}$

So the resulting concordance matrix is:

$c=\left[\begin{array}{ccccc}- & c_{12} & c_{13} & \cdots & c_{1 n} \\ c_{21} & - & c_{23} & \cdots & c_{2 n} \\ \cdots & & & & \\ c_{m 1} & c_{m 2} & c_{m 3} & \cdots & -\end{array}\right]$

Discordance

To determine the value of the elements in the discordance matrix is to divide the maximum of the difference of the criterion value included in the discordance subset by the maximum difference in the value of all the existing criteria, metaphysically is:

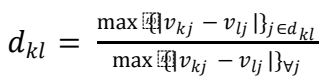

Furthermore, the discordance matrix is obtained: $d=\left[\begin{array}{ccccc}- & d_{12} & d_{13} & \cdots & d_{1 n} \\ d_{21} & - & d_{23} & \cdots & d_{2 n} \\ \cdots & & & & \\ d_{m 1} & d_{m 2} & d_{m 3} & \cdots & -\end{array}\right]$

Step 5: Determine the dominant matrix of concordance and discordance

Concordance

Calculate the dominant matrix of concordance with the following equation:

$\underline{c}=\frac{\sum_{k=1}^{n} \Sigma_{l=1}^{n} c_{k l}}{m \cdot(m-1)}$

And the value of each element of the matrix $\mathbf{F}$ as the dominant matrix of concordance is determined as the following equation:

$f_{k l}=1$, if $c_{k l} \geq \underline{c}$ and $f_{k l}=0$, if $c_{k l}<\underline{c}$

Discordance

To construct the dominant matrix of discordance also use the help of threshold value, that is with the following equation:

$\underline{d}=\frac{\sum_{k=1}^{n} \Sigma_{l=1}^{n} d_{k l}}{m(m-1)}$

And the value of each element for the matrix $\boldsymbol{G}$ as the dominant matrix of discordance is determined as the following equation:

$g_{k l}=1$, if $d_{k l} \geq \underline{d}$ and $g_{k l}=0$, if $d_{k l}<\underline{d}$

Step 6: Determine the aggregate dominance matrix.

The next step is to determine the aggregate dominance matrix as the matrix $\boldsymbol{E}$, each element being a multiplication of the matrix element $f$ with the matrix element $g$, as the following equation:

$e_{k l}=f_{k l} \times g_{k l}$

From the equation it produces a matrix $e$ provides a sequence of options from each alternative, ie when $\boldsymbol{E}_{\boldsymbol{k}}=1$ then alternate $\mathrm{A}_{k}$ is a better option than $\mathrm{A}_{r}$ so the line is in the matrix $\boldsymbol{E}$ which has the amount $\boldsymbol{E}_{\boldsymbol{k} l}=1$ at least can be eliminated. Thus the best alternative is that which dominates other alternatives.

\section{DESIGN OF RESEARCH}

\subsection{Feature Dataset}

Materials to be used in this study include data from survey results and interviews from experts for determining the location of disaster evacuation and the value of the criteria weight and data from the Regulation of the Head of National Agency for Disaster Management Number 14 of 2010. The data obtained can be seen in Table I.

Table 1. Location criteria and weight for tsunami disaster evacuation

\begin{tabular}{|l|l|l|l|l|}
\hline \multirow{2}{*}{ Code } & \multirow{2}{*}{ Criteria } & \multicolumn{2}{|l|}{ Weight } \\
\cline { 3 - 5 } & & $\begin{array}{l}\text { Expert } \\
\mathbf{1}\end{array}$ & $\begin{array}{l}\text { Expert } \\
\mathbf{2}\end{array}$ & $\begin{array}{l}\text { Expert } \\
\mathbf{3}\end{array}$ \\
\hline C1 & Location & 5 & 4 & 5 \\
\hline C2 & Population & 5 & 3 & 3 \\
\hline C3 & Accessibility & 5 & 5 & 5 \\
\hline C4 & Topographic area & 5 & 5 & 5 \\
\hline
\end{tabular}




\begin{tabular}{|l|l|l|l|l|}
\hline \multirow{2}{*}{ Code } & \multirow{2}{*}{ Criteria } & \multicolumn{2}{|l|}{ Weight } \\
\cline { 3 - 5 } & $\begin{array}{l}\text { Expert } \\
\mathbf{1}\end{array}$ & $\begin{array}{l}\text { Expert } \\
\mathbf{2}\end{array}$ & $\begin{array}{l}\text { Expert } \\
\mathbf{3}\end{array}$ \\
\hline C5 & $\begin{array}{l}\text { Building } \\
\text { Orientation }\end{array}$ & 3 & 3 & 4 \\
\hline C6 & Space Utilization & 5 & 5 & 5 \\
\hline
\end{tabular}

To know the level given for each object, which is from the criteria that have a great influence on the other ratios for that scale using 1 to 5 and as follows:

$1=$ Very ineffective

$2=$ Ineffective

$3=$ Neutral

$4=$ Effective

$5=$ Very effective

\subsection{System Framework}

The system framework is a general overview of the information system design to be created and depicted in a flow or data consisting of inputs, processes and outputs, explaining how the system works when executed by the user in an effort to provide information for determining the location of the tsunami disaster evacuation using the ELECTRE method Outline of the system framework can be seen in (Figure 1).

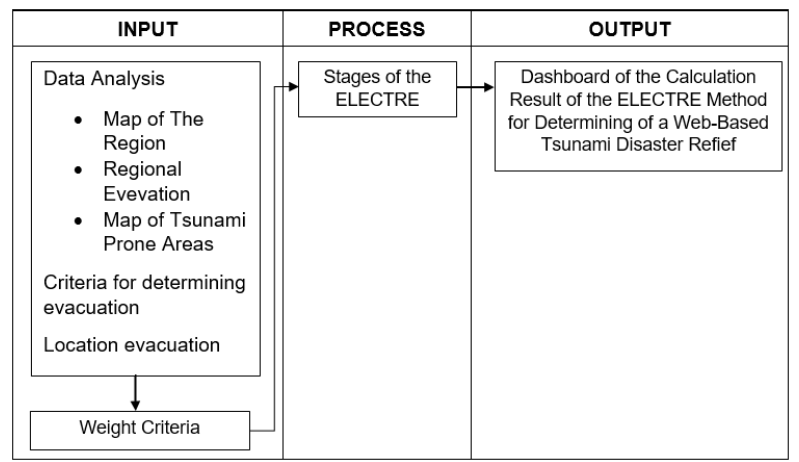

Fig 1: Information system framework

\section{RESULT AND ANALYSIS}

To achieve the objectives of this study discussed earlier, there are several stages in the application of the ELECTE method to be performed. The stages are divided into several processes including, normalizing the decision matrix, weighting the normalized matrix, determining the set of concordance and discordance index, calculating concordance and discordance matrix, determining the dominant matrix of concordance and discordance, determining aggregate dominance matrix.

\subsection{Application of the ELECTRE Method}

Criteria affecting the selection of evacuation sites for tsunami disaster, which are required in the application of the ELECTRE method and alternative sites for evacuation sites. Criteria affecting site selection are determined by Table I and for determining the location of evacuation points conducted by the Regional Disaster Management Agency of Bitung City and the results can be seen in Table 2 .
Table 2. Location criteria and weight for tsunami disaster evacuation

\begin{tabular}{|l|l|}
\hline Code & Location \\
\hline L1 & Terminal Tangkoko \\
\hline L2 & Stadium Dua Sudara \\
\hline L3 & Firing range \\
\hline L4 & SMP Negeri 12 Bitung \\
\hline L5 & Field SMK Dharma Bakti Bitung \\
\hline L6 & Field SMK Negeri 2 Bitung \\
\hline L7 & Container Terminal \\
\hline L8 & Field Kakenturan 1 \\
\hline L9 & Field Kakenturan 2 \\
\hline L10 & Winenet Market \\
\hline
\end{tabular}

To perform the calculation, it is necessary to adjust the table between the location and the criteria and for the conformity value of each - each criterion can be seen in Table 3 by using the value from BPBD City Bitung.

Table 3. Match rating of each location on each criterion

\begin{tabular}{|l|l|l|l|l|l|l|}
\hline \multirow{2}{*}{ Location } & \multicolumn{6}{|l}{ Criteria } \\
\cline { 2 - 7 } & C1 & C2 & C3 & C4 & C5 & C6 \\
\hline L1 & 5 & 4 & 5 & 5 & 4 & 5 \\
\hline L2 & 5 & 4 & 5 & 5 & 5 & 5 \\
\hline L3 & 4 & 5 & 4 & 4 & 4 & 4 \\
\hline L4 & 4 & 5 & 5 & 3 & 4 & 4 \\
\hline L5 & 4 & 5 & 4 & 4 & 4 & 4 \\
\hline L6 & 4 & 4 & 5 & 4 & 4 & 4 \\
\hline L7 & 5 & 4 & 4 & 4 & 4 & 4 \\
\hline L8 & 4 & 5 & 4 & 4 & 4 & 4 \\
\hline L9 & 4 & 4 & 4 & 4 & 4 & 4 \\
\hline L10 & 4 & 4 & 4 & 4 & 4 & 4 \\
\hline
\end{tabular}

The next step determines the weight of each criterion and the criteria weight is determined from the BPBD of Bitung City as shown in Table 1 and the average is searched so that it creates a new value. The value can be seen in Figure 2 which is the weight of the average value of each criterion.

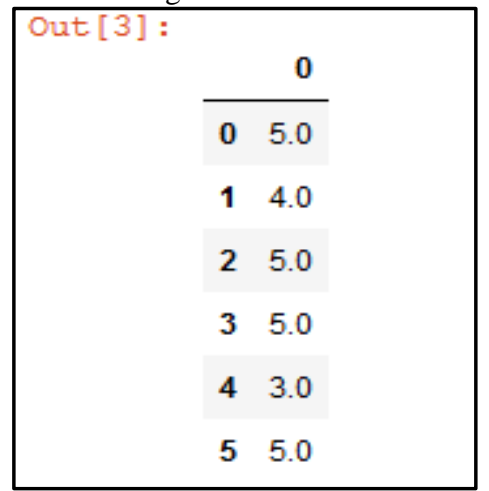

Fig 2: Weight criteria 
The next step performs calculations using the ELECTRE method and for this calculation phase using Python programming language, the stages are as follows:

Step 1: Normalize the decision matrix.

For normalization of matrix by using equation (1), so obtained matrix $\mathbf{R}$ from result of normalization and for matrix $\mathbf{R}$ can be seen in Figure 3.

\begin{tabular}{|c|c|c|c|c|c|c|c|}
\hline \multicolumn{8}{|l|}{ Out [40]: } \\
\hline & 0 & 0.4 & 0.3 & 0.4 & 0.4 & 0.3 & 0.4 \\
\hline & 1 & 0.4 & 0.3 & 0.4 & 0.4 & 0.4 & 0.4 \\
\hline & 2 & 0.3 & 0.4 & 0.3 & 0.3 & 0.3 & 0.3 \\
\hline & 3 & 0.3 & 0.4 & 0.4 & 0.2 & 0.3 & 0.3 \\
\hline & 4 & 0.3 & 0.4 & 0.3 & 0.3 & 0.3 & 0.3 \\
\hline & 5 & 0.3 & 0.3 & 0.4 & 0.3 & 0.3 & 0.3 \\
\hline & 6 & 0.4 & 0.3 & 0.3 & 0.3 & 0.3 & 0.3 \\
\hline & 7 & 0.3 & 0.4 & 0.3 & 0.3 & 0.3 & 0.3 \\
\hline & 8 & 0.3 & 0.3 & 0.3 & 0.3 & 0.3 & 0.3 \\
\hline & 9 & 0.3 & 0.3 & 0.3 & 0.3 & 0.3 & 0.3 \\
\hline
\end{tabular}

Fig 3: Normalize matrix

Step 2: Weighing on a normalized matrix.

After normalization, each column of the matrix $\mathbf{R}$ is multiplied by the weight of the criterion by using equation (3) and the form of the weighted result on the normalized matrix can be seen in Figure 4.

\begin{tabular}{|c|c|c|c|c|c|c|c|}
\hline \multicolumn{8}{|l|}{ Out [41]: } \\
\hline \multirow{10}{*}{ Out [41] : } & 0 & 1.8282 & 1.1429 & 1.7857 & 1.9118 & 0.9231 & 1.8738 \\
\hline & 1 & 1.8282 & 1.1429 & 1.7857 & 1.9118 & 1.1538 & 1.8738 \\
\hline & 2 & 1.4625 & 1.4286 & 1.4286 & 1.5294 & 0.9231 & 1.4991 \\
\hline & 3 & 1.4625 & 1.4286 & 1.7857 & 1.1471 & 0.9231 & 1.4991 \\
\hline & 4 & 1.4625 & 1.4286 & 1.4286 & 1.5294 & 0.9231 & 1.4991 \\
\hline & 5 & 1.4625 & 1.1429 & 1.7857 & 1.5294 & 0.9231 & 1.4991 \\
\hline & 6 & 1.8282 & 1.1429 & 1.4286 & 1.5294 & 0.9231 & 1.4991 \\
\hline & 7 & 1.4625 & 1.4286 & 1.4286 & 1.5294 & 0.9231 & 1.4991 \\
\hline & 8 & 1.4625 & 1.1429 & 1.4286 & 1.5294 & 0.9231 & 1.4991 \\
\hline & 9 & 1.4625 & 1.1429 & 1.4286 & 1.5294 & 0.9231 & 1.4991 \\
\hline
\end{tabular}

Fig 4: Weighted on a normalized matrix

Step 3: Determine the set of concordance and discordance index.

This stage is done by comparing the value of each criterion of an alternative with the other alternatives one by one, starting with the first alternative with the second, the first with the third alternative, and so on, if the value of the comparable alternative is greater than the comparison, the index is used as an index concordance exists when it is smaller it will be used as a discordance index, for example following the first alternative comparison with the second alternative:

Concordance Index:

$C_{12}=\{2,5,6\}$

$C_{13}=\{2,3,5,6\}$

$C_{14}=\{2,3,5,6\}$

$\mathrm{C}_{\ldots .}=\ldots$

Discordance Index:

$D_{12}=\{1,3,4\}$

$D_{13}=\{1,4\}$

$D_{14}=\{1,4\}$

$D_{\ldots .}=\ldots$

For the results of the system can be seen in Figure 5 and Figure 6.

\begin{tabular}{|crrrrrrr|}
\hline Out [42] : & & & & & & & \\
& & 0 & 1 & 2 & 3 & 4 & 5 \\
\cline { 2 - 5 } & $\mathbf{0}$ & True & True & True & True & True & True \\
& $\mathbf{1}$ & True & True & True & True & True & True \\
& $\mathbf{2}$ & True & True & True & True & True & True \\
& 3 & True & True & True & False & True & True \\
& 4 & True & True & True & True & True & True \\
& 5 & True & True & True & True & True & True \\
6 & True & True & True & True & True & True \\
7 & True & True & True & True & True & True \\
$\mathbf{8}$ & True & True & True & True & True & True \\
$\mathbf{9}$ & False & False & False & False & False & False \\
& & & & & & &
\end{tabular}

Fig 5: Concordance index

\begin{tabular}{|crrrrrrr}
\hline Out [43] : & & & & & & \\
& & 0 & 1 & 2 & 3 & 4 & 5 \\
\hline & 0 & False & False & False & False & False & False \\
& 1 & False & False & False & False & False & False \\
& 2 & False & False & False & False & False & False \\
& 3 & False & False & False & True & False & False \\
4 & False & False & False & False & False & False \\
5 & False & False & False & False & False & False \\
6 & False & False & False & False & False & False \\
7 & False & False & False & False & False & False \\
8 & False & False & False & False & False & False \\
9 & False & False & False & False & False & False \\
\hline
\end{tabular}

Fig 6: Discordance index

Step 4: Calculate concordance and discordance matrices

a. Calculating the concordance matrix (Matrix C) is done by summing the criteria weight based on the concordance 
index. The calculation results will be in the form of a matrix as in Figure 7.

\begin{tabular}{|c|c|c|c|c|c|c|c|c|c|c|c|}
\hline \multicolumn{12}{|l|}{ Out [44]: } \\
\hline & 0 & 0.0 & 24.0 & 23.0 & 23.0 & 23.0 & 27.0 & 27.0 & 23.0 & 27.0 & 27.0 \\
\hline & 1 & 27.0 & 0.0 & 23.0 & 23.0 & 23.0 & 27.0 & 27.0 & 23.0 & 27.0 & 27.0 \\
\hline & 2 & 7.0 & 4.0 & 0.0 & 22.0 & 27.0 & 22.0 & 22.0 & 27.0 & 27.0 & 27.0 \\
\hline & 3 & 12.0 & 9.0 & 22.0 & 0.0 & 22.0 & 22.0 & 17.0 & 22.0 & 22.0 & 22.0 \\
\hline & 4 & 7.0 & 4.0 & 27.0 & 22.0 & 0.0 & 22.0 & 22.0 & 27.0 & 27.0 & 27.0 \\
\hline & 5 & 12.0 & 9.0 & 23.0 & 23.0 & 23.0 & 0.0 & 22.0 & 23.0 & 27.0 & 27.0 \\
\hline & 6 & 12.0 & 9.0 & 23.0 & 18.0 & 23.0 & 22.0 & 0.0 & 23.0 & 27.0 & 27.0 \\
\hline & 7 & 7.0 & 4.0 & 27.0 & 22.0 & 27.0 & 22.0 & 22.0 & 0.0 & 27.0 & 27.0 \\
\hline & 8 & 7.0 & 4.0 & 23.0 & 18.0 & 23.0 & 22.0 & 22.0 & 23.0 & 0.0 & 27.0 \\
\hline & 9 & 7.0 & 4.0 & 23.0 & 18.0 & 23.0 & 22.0 & 22.0 & 23.0 & 27.0 & 0.0 \\
\hline
\end{tabular}

Fig 7: Concordance matrix

b. Calculating the discordance matrix (Matrix D) is performed by finding the highest value of the difference in the value of the comparison based on the discordance index is given the highest value of the comparison of the entire criteria index. The calculation results will be in the form of a matrix as in Figure 8

\begin{tabular}{|c|c|c|c|c|c|c|c|c|c|c|c|}
\hline \multicolumn{12}{|l|}{ Out [45] : } \\
\hline & 0 & 0.0 & 1.0 & 0.75 & 0.37 & 0.75 & 0.00 & 0.0 & 0.75 & 0.0 & 0.0 \\
\hline & 1 & 0.0 & 0.0 & 0.75 & 0.37 & 0.75 & 0.00 & 0.0 & 0.75 & 0.0 & 0.0 \\
\hline & 2 & 1.0 & 1.0 & 0.00 & 0.93 & 0.00 & 1.00 & 1.0 & 0.00 & 0.0 & 0.0 \\
\hline & 3 & 1.0 & 1.0 & 1.00 & 0.00 & 1.00 & 1.00 & 1.0 & 1.00 & 1.0 & 1.0 \\
\hline & 4 & 1.0 & 1.0 & 0.00 & 0.93 & 0.00 & 1.00 & 1.0 & 0.00 & 0.0 & 0.0 \\
\hline & 5 & 1.0 & 1.0 & 0.80 & 0.75 & 0.80 & 0.00 & 1.0 & 0.80 & 0.0 & 0.0 \\
\hline & 6 & 1.0 & 1.0 & 0.78 & 0.93 & 0.78 & 0.98 & 0.0 & 0.78 & 0.0 & 0.0 \\
\hline & 7 & 1.0 & 1.0 & 0.00 & 0.93 & 0.00 & 1.00 & 1.0 & 0.00 & 0.0 & 0.0 \\
\hline & 8 & 1.0 & 1.0 & 1.00 & 0.93 & 1.00 & 1.00 & 1.0 & 1.00 & 0.0 & 0.0 \\
\hline & 9 & 1.0 & 1.0 & 1.00 & 0.93 & 1.00 & 1.00 & 1.0 & 1.00 & 0.0 & 0.0 \\
\hline
\end{tabular}

\section{Fig 8: Concordance matrix}

Step 5: Determine the dominant matrix of concordance and discordance.

To determine the dominant matrix of concordance (matrix F) and matrix of discordance (matrix G), it is necessary to determine the threshold value of each matrix and then compare the value of concordance matrix (matrix C) with the concordance matrix value, as well as the determination of the dominant matrix of discordance by determining the discordance matrix threshold value.

a. Determining the threshold value of the concordance matrix with equation (10) and the result can be seen in Figure 9.

\section{Threshold Value C 20.9666666666666665}

Fig 9: Threshold value $\underline{\mathrm{c}}$

The dominant matrix element of concordance is generated by comparing the value of the concordance matrix (Matrix C) to the threshold value of the concordance matrix and to determine it by using equation (11) and the results can be seen in Figure 10

\begin{tabular}{|crrrrrrrrrrr|}
\hline Out [48] : & & 0 & 1 & 2 & 3 & 4 & 5 & 6 & 7 & 8 & 9 \\
\cline { 2 - 3 } & 0 & False & True & True & True & True & True & True & True & True & True \\
& $\mathbf{1}$ & True & False & True & True & True & True & True & True & True & True \\
& 2 & False & False & False & True & True & True & True & True & True & True \\
& 3 & False & False & True & False & True & True & False & True & True & True \\
& 4 & False & False & True & True & False & True & True & True & True & True \\
& 5 & False & False & True & True & True & False & True & True & True & True \\
6 & False & False & True & False & True & True & False & True & True & True \\
7 & False & False & True & True & True & True & True & False & True & True \\
8 & False & False & True & False & True & True & True & True & False & True \\
9 & False & False & True & False & True & True & True & True & True & False \\
\hline
\end{tabular}

Fig 10: Dominant concordance matrix

b. Determining the threshold value of the concordance matrix with equation (12) and the result can be seen in Figure 11.

$$
\begin{aligned}
& \text { Threshold Value D } \\
& 0.6478137630362827
\end{aligned}
$$

Fig 11: Threshold value $\underline{\mathrm{d}}$

The dominant matrix element of discordance is generated by comparing the value on the discordance matrix (Matrix D) to the discordance matrix threshold value and for determining it by using equation (13) and the results can

\begin{tabular}{|c|c|c|c|c|c|c|c|c|c|c|c|}
\hline \multirow[t]{11}{*}{ Out [49] : } & & 0 & 1 & 2 & 3 & 4 & 5 & 6 & 7 & 8 & 9 \\
\hline & 0 & False & True & True & False & True & False & False & True & False & False \\
\hline & 1 & False & False & True & False & True & False & False & True & False & False \\
\hline & 2 & True & True & False & True & False & True & True & False & False & False \\
\hline & 3 & True & True & True & False & True & True & True & True & True & True \\
\hline & 4 & True & True & False & True & False & True & True & False & False & False \\
\hline & 5 & True & True & True & True & True & False & True & True & False & False \\
\hline & 6 & True & True & True & True & True & True & False & True & False & False \\
\hline & 7 & True & True & False & True & False & True & True & False & False & False \\
\hline & 8 & True & True & True & True & True & True & True & True & False & False \\
\hline & 9 & True & True & True & True & True & True & True & True & False & False \\
\hline
\end{tabular}
be seen in Figure 12.

Fig 12: Dominant discordance matrix

Step 6: Determine the aggregate dominance matrix.

The dominant aggregate matrix is obtained from the dominant matrix of concordance (matrix F) with the dominant matrix of discordance (matrix $\mathrm{G}$ ), so that the dominant aggregate matrix is obtained as follows: 


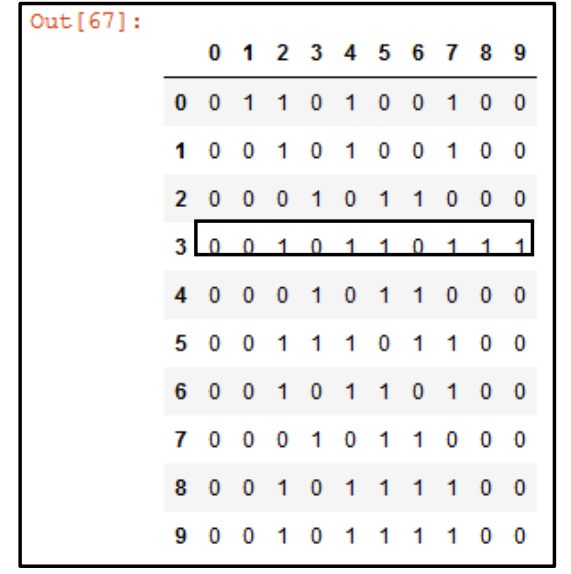

Fig 13: The shape of the matrix $E$

From the dominant aggregate matrix above can be determined the best alternative location, that is the alternative with the total number of $\boldsymbol{E}_{k l}=1$ will be selected as the best alternative, that is the 4th location with the number of $\boldsymbol{E}_{k l}=1$ counted 6 .

\subsection{System Trial and Validation}

The process of system testing is done by performing system validation by ELECTRE method. Validation of this system using five alternatives and 5 criteria. The value used to perform system validation can be seen in Table 4 and for the value of $\mathbf{W}=[5,5,5,5,5]$

Table 4. Alternative to Criteria with Random Values in the First Test

\begin{tabular}{|l|l|l|l|l|l|}
\hline \multirow{2}{*}{ Alternative } & \multicolumn{4}{|l|}{ Criteria } \\
\cline { 2 - 6 } & C1 & C2 & C3 & C4 & C5 \\
\hline A1 & 5 & 5 & 5 & 5 & 5 \\
\hline A2 & 4 & 4 & 4 & 4 & 4 \\
\hline A3 & 3 & 3 & 3 & 3 & 3 \\
\hline A4 & 2 & 2 & 2 & 2 & 2 \\
\hline A5 & 1 & 1 & 1 & 1 & 1 \\
\hline
\end{tabular}

By looking at Table 4 above it can be ascertained under Alternative 1 that has a better value and seen in the system that the results are the same and the results can be seen in Figure 14.

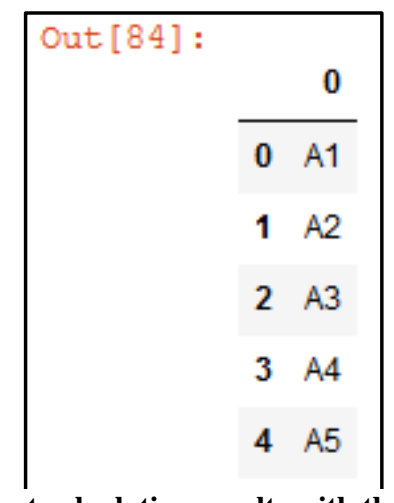

Fig 14: The first calculation results with the ELECTRE method of the system

In this second phase test still using the same criteria weight value is 5 and for the alternative value against the criteria can be seen in Table 5 .
Table 5. Alternatives to criteria with value of activity in second testing

\begin{tabular}{|l|l|l|l|l|l|}
\hline \multirow{2}{*}{ Alternative } & \multicolumn{4}{|l|}{ Criteria } \\
\cline { 2 - 6 } & C1 & C2 & C3 & C4 & C5 \\
\hline A1 & 3 & 3 & 3 & 3 & 3 \\
\hline A2 & 4 & 4 & 4 & 4 & 4 \\
\hline A3 & 5 & 5 & 5 & 5 & 5 \\
\hline A4 & 2 & 2 & 2 & 2 & 2 \\
\hline A5 & 1 & 1 & 1 & 1 & 1 \\
\hline
\end{tabular}

By looking at Table 5 it can be ascertained that Alternative 3 has a better value, and can also be seen in the system that the results are the same and the results can be seen in Figure 15

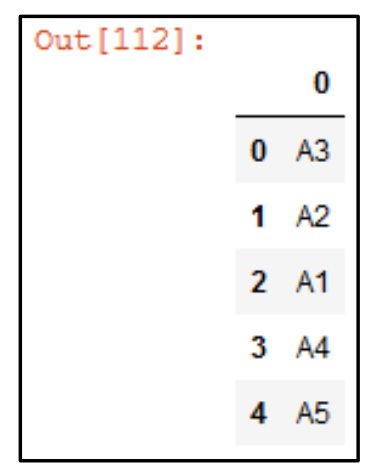

Fig 15: The second calculation results with the ELECTRE method of the system

With the results of testing on the system made it can be concluded under the method of ELECTRE has succeeded in accordance with the steps of the method.

\section{CONCLUSION}

The system for selecting the tsunami disaster evacuation site using the ELECTRE method has been established to support BPBD parties in the decision making that have been implemented in this system. The results of calculations in the ELECTRE method can be used as a recommendation for the selection of tsunami evacuation sites. The advantages of the ELECTRE method can provide the best alternative decision solutions in decision making with many criteria. Based on the calculation, states that the location of SMP Negeri 12 Bitung is the best location with the number of $\mathbf{E}_{\mathrm{kl}}=1$ as much as 6 .

The results of this study can be developed by becoming a Geographic Information System (GIS) in order to see the position of the region if the tsunami occurred, the height of the area to be used the location of the point of evacuation, and the ingredients affected.

\section{ACKNOWLEDGMENTS}

Thanks to all those who have assisted in this field of research. Especially to the Regional Disaster Management Agency of Bitung city because it has been willing to assist in providing data to conduct this research

\section{REFERENCES}

[1] BMKG, Pedoman Pelayanan Peringatan Dini Tsunami InaTEWS, Edisi Kedu. 2012.

[2] A. Teixeira de Almeida, "Multicriteria decision model for outsourcing contracts selection based on utility function and ELECTRE method," Comput. Oper. Res., 
vol. 34, no. 12, pp. 3569-3574, 2007.

[3] G. Łaska, "Wind Energy and Multi-criteria Analysis in Making Decisions on the Location of Wind Farms," Procedia Eng., vol. 182, pp. 418-424, 2017.

[4] L. Botti and N. Peypoch, "Multi-criteria ELECTRE method and destination competitiveness," Tour. Manag. Perspect., vol. 6, pp. 108-113, 2013.

[5] S. P. Rodrigues, L. A. Costa, D. Produc, and U. Minho, "Comparing AHP and ELECTRE I for prioritizing software requirements," 2015.

[6] P. Kumar, R. K. Singh, and K. Kharab, "A comparative analysis of operational performance of Cellular Mobile Telephone Service Providers in the Delhi working area using an approach of fuzzy ELECTRE," Appl. Soft Comput. J., vol. 59, pp. 438-447, 2017.

[7] S. Birgun and E. Cihan, "Supplier selection process using ELECTRE method," in 2010 IEEE International Conference on Intelligent Systems and Knowledge Engineering, 2010, pp. 634-639.

[8] L. Marlinda, T. Baidawi, Y. Durachman, and
Akmaludin, "A multi-study program recommender system using ELECTRE multicriteria method," 2017 5th Int. Conf. Cyber IT Serv. Manag. CITSM 2017, pp. $1-5,2017$.

[9] M. Beynon, "Fuzzy Outranking Methods Including Fuzzy PROMETHEE," in Handbook of Research on Fuzzy Information Processing in Databases, 1st ed., Hershey • New York: IGI Global, 2008, pp. 784-804.

[10] E. Triantaphyllou, Multi-criteria Decision Making Methods: A Comparative Study, 44th ed., vol. 44. Boston, MA: Springer US, 2000.

[11] M. Rogers, M. Bruen, and L.-Y. Maystre, ELECTRE and Decision Support. Boston, MA: Springer US, 2000.

[12] D. Simić, J. Gajić, V. Ilin, V. Svirčević, and S. Simić, "A Hybrid Bio-inspired ELECTRE Approach for Decision Making in Purchasing Agricultural Equipment," vol. 8073, J.-S. Pan, M. M. Polycarpou, M. Woźniak, A. C. P. L. F. de Carvalho, H. Quintián, and E. Corchado, Eds. Berlin, Heidelberg: Springer Berlin Heidelberg, 2016, pp. 283-295. 